\title{
Les définitions de l'énoncé (vākya) dans la tradition sanskrite : entre grammaire et exégèse
}

\section{INTRODUCTION : UNE "SCIENCE DE L'ÉNONCÉ " ? ${ }^{1}$}

1.1. Selon la répartition des disciplines d'érudition (śāstra) d'expression sanskrite courante dans l'Inde médiévale, c'est à l'exégèse du Veda (Mìmāmsā) que revient en principe l'étude de l'énoncé $(v \bar{a} k y a)^{2}$. Toute entière consacrée à l'interprétation des textes védiques, l'exégèse se qualifie comme la "science de l'énoncé » (vākyaśāstra) par excellence, et c'est à ce titre que son expertise s'étend, par voie de conséquence, à l'analyse des énoncés profanes ${ }^{3}$. Elle se situe de ce fait au centre d'une organisation tripartite où la grammaire (Vyākarana) traite, en amont, de la dérivation des mots (pada) du sanskrit alors que, en aval, la logique ou dialectique (Nyāya / Tarka) étudie les moyens de connaissance valide (pramāna) - perception, inférence, etc. - s'appliquant aux objets extralinguistiques. Trois sciences (śāstra), donc, pour trois domaines distincts : la langue sanskrite, les énoncés concrets composés dans cette langue et la connaissance du monde auquel ils font ultimement référence. Armé de ce triple organon, le lettré "versé dans [les sciences] du mot, de l'énoncé et du moyen de connaissance valide » (padavākyapramānajña) peut aborder sur une base solide, selon l'idée

\footnotetext{
1. Nous remercions S.L.P. Anjaneya Sarma et Vincenzo Vergiani, avec qui nous avons pu lire et discuter la plupart des passages grammaticaux ici commentés durant l'été 2014 à Pondichéry, ainsi que les participants au présent volume pour leurs remarques et critiques aux divers stades de la rédaction de cet article.

2. Nous emploierons dans cet article les deux termes «phrase » et « énoncé » comme synonymes. La plupart des travaux disponibles sur le sujet étant en anglais, leurs auteurs n'opèrent pas cette distinction et traduisent uniformément vākya par « sentence » (plus rarement par «utterance »).

3. Sur l'utilisation par les spécialistes de théorie littéraire et dramaturgique de principes élaborés par la Mìmāmsā pour l'interprétation des textes védiques, voir McCrea (2008) et David (2016).
} 
indienne, toutes les autres disciplines érudites, religieuses (théologie, rituel, etc.) ou profanes (médecine, astronomie, poétique, etc.).

1.2. Dans les faits, cette division idéale des tâches masque une réalité beaucoup plus complexe. Loin de se compléter au sein d'un ensemble parfaitement harmonieux, les trois composantes de ce qui s'apparente en surface à une forme de trivium évoluent en effet à des rythmes très différents ${ }^{4}$ et suivant une logique qui leur est propre. Toutes trois présentent, par ailleurs, une tendance totalisante qui les incite à couvrir, par-delà leur domaine d'expertise, l'intégralité du champ du savoir. Sur la question qui nous occupe, bien que l'énoncé tombe théoriquement hors du domaine de juridiction de la grammaire ou de la logique, en pratique aucune de ces deux sciences ne s'y est montrée indifférente. Si ce n'est qu'assez tardivement (et, peut-on penser, largement sous l'influence de la Mìmāms $\bar{a}$ ) que la logique aborde la question, la théorie de l'énoncé revêt pour la tradition grammaticale ancienne une importance certaine, qui ne fait que s'accroître après l'apparition de l'exégèse comme science autonome dans les premiers siècles de notre ère.

1.3. C'est aux premières interactions entre exégèse et grammaire sur la question des définitions (lakșana) de l'énoncé qu'est consacrée la présente étude. Après un rappel du problème des définitions du vākya dans la tradition grammaticale ancienne $(\S 2)$, on examinera l'état de cette question au $\mathrm{V}^{\mathrm{e}}$ siècle de notre ère dans le Vākyapadīya ("Traité du Mot et de la Phrase », désormais VP) du grammairien Bhartrhari (450-520 ?) (§ 3). Euvre unique en son genre et premier monument marquant le renouveau de la tradition pāninéenne au premier millénaire, le Vākyapadīya est également le premier texte grammatical sanskrit à consacrer une section entière à l'énoncé, le Vākyakānḍa ("Section sur l'énoncé »), en près de cinq cents stances (soit environ un quart de l'œuvre). Nous nous efforcerons ici de montrer en quoi l'introduction par Bhartrhari d'un certain nombre de concepts empruntés à l'exégèse lui permet de renouveler en profondeur le traitement de la question du vākya par ses prédécesseurs, en droite ligne avec sa conception de l'énoncé comme unité indivise (akhanda), irréductible à la combinaison de ses parties.

\section{L'ÉNONCÉ DANS LA GRAMMAIRE PĀNINÉENNE ANCIENNE}

2.1. Lorsque Bhartrhari se saisit de la question des définitions du vākya, celle-ci a déjà une histoire longue et mouvementée dans la tradition pāṇinéenne, documentée dans l'œuvre des « Trois Sages » (Trimuni) de la grammaire ancienne :

\footnotetext{
4. Pour ne prendre qu'un exemple, le Grand Commentaire (Mahābhāsya) de Patañjali sur l'Asțādhyāȳ («Les Huit Livres ») de Pānini, commentaire que l'on peut considérer comme le texte fondateur de la tradition grammaticale sanskrite, pourrait dater du $\mathrm{II}^{\mathrm{e}}$ siècle AEC alors que son plus proche équivalent pour l'exégèse védique, le Commentaire sur les Aphorismes de l'Exégèse (Mīmāmsāsūtrabhāṣa) de Śabarasvāmin a probablement été rédigé au $\mathrm{IV}^{\mathrm{e}}$ ou $\mathrm{V}^{\mathrm{e}}$ siècle $\mathrm{EC}$, soit environ six siècles plus tard.
} 
Pāninini (IV $\mathrm{e}$ s. AEC), Kātyāyana (III ${ }^{\mathrm{e}}$ s. AEC) et Patañjali (II ${ }^{\mathrm{e}}$ s. AEC). La question telle qu'elle apparaît chez ces auteurs ayant déjà fait l'objet de plusieurs bonnes études ${ }^{5}$, nous nous bornerons ici à rappeler les principales données du problème.

2.2. C'est d'abord de manière négative que l'énoncé se manifeste aux grammairiens anciens et de telle sorte qu'il se trouve exclu du champ de la grammaire, quand bien même celle-ci se doit comme on va le voir (infra § 2.3) de le théoriser pour assurer son bon fonctionnement. Ce qu'exprime l'énoncé, c'est avant tout ce qui est compris par les acteurs d'une situation d'interlocution mais résiste à la formalisation grammaticale. Considérons, à titre d'exemple, l'aphorisme 2.3.46 de l'Aștāidhyāỹ (A), dans lequel Pāninini énumère les valeurs justifiant l'usage des désinences du premier cas (prathamā [vibhaktih]), le nominatif (Nom):

\section{A 2.3.46 \\ prātipadikārthalingaparimānavacanamātre prathamā}

Les désinences du premier cas (i.e. le nominatif) valent s'il s'agit d'exprimer (a) le sens du thème nominal, (b) le genre grammatical, (c) la mesure, (d) le nombre grammatical (à moins que lesdites notions ne soient déjà exprimées par ailleurs - A 2.3.1 anabhihite) ${ }^{6}$.

Les premiers commentateurs sur cet aphorisme rencontrent une difficulté s'agissant d'expliquer l'usage du nominatif dans le cas d'une phrase purement nominale, comme dans l'exemple suivant :

\section{vīrah purusah}

L'homme (vira-Nom) [est] courageux (purusa-Nom).

Ce type de phrases, extrêmement fréquent en sanskrit, consiste en effet en l'apposition de deux termes au nominatif sans adjonction d'une copule. Or, il est manifeste qu'un tel énoncé n'exprime pas seulement le sens des deux bases nominales, le masculin et le singulier, mais aussi ce que les grammairiens nomment la " coréférentialité » (sāmānādhikaranya) des deux termes au nominatif, à savoir le fait qu'ils correspondent à une seule et même entité (l'homme courageux) via une relation de qualifiant à qualifié (viśesyaviśeșanabhāva). C'est précisément l'excès (adhikya) que représente la coréférentialité par rapport aux valeurs du nominatif énumérées par Pānini qui suscite l'interrogation de Kātyāyana : n'estil pas nécessaire d'élargir cet éventail de valeurs de manière à pouvoir inclure la coréférentialité ? La première de ses « Explications » (Vārttika) sur cet aphorisme propose $\mathrm{d}^{\prime}$ abord une réponse positive à cette question :

Vārttika 1 ad A 2.3.46

prātipadikārthalingaparimānavacanamātre prathamālakṣane padasāmānādhikaranya upasaṃkhȳ̄nam, adhikatvāt

Il convient d'ajouter à la définition du premier cas (i.e. le nominatif), à savoir que <les désinences du premier cas valent s'il s'agit d'exprimer (a) le sens du thème

5. Voir en particulier Raja (1976), Cardona (1983) et Deshpande (1987, 1991).

6. Sauf indication contraire, la traduction des sūtra de Pāninini est celle de Renou (1966). 
nominal, etc.> (A 2.3.46), la coréférentialité des mots [au nominatif], car elle est en excès [par rapport aux valeurs mentionnées].

Telle n'est cependant pas la décision finale de Kātyāyana, qui précise aussitôt :

Vārttika 2 ad A 2.3 .46

na vā, vākyārthatvāt

Ou plutôt, non : [un tel ajout n'est pas nécessaire] car il s'agit de l'objet de l'énoncé (vākyārtha).

Selon Kātyāyana, la coréférentialité des deux termes n'est donc pas directement le fruit de l'usage du nominatif, mais plutôt de la juxtaposition de deux mots au nominatif au sein d'un même énoncé. Ainsi, il n'est pas nécessaire selon sa position finale d'amender l'aphorisme pāninéen. C'est Patañjali qui, dans la section correspondante du Mahābhāṣya, pose de manière définitive l'équivalence

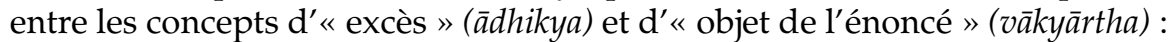

Mahābhāṣya ad A 2.3.46 (vol. 1, p. 462, 1. 4-5)

yad atrādhikyam, vākyārthah sah

Ce qui se trouve ici en excès, $c^{\prime}$ est cela l'objet de l'énoncé ${ }^{7}$.

L'objet de l'énoncé, " en excès » par rapport à celui de tel ou tel thème ou désinence, n'a donc pas lieu d'être formalisé par la grammaire. La production de sens revenant en propre à l'énoncé est, en effet, sans conséquence sur le processus de dérivation des mots lui-même, à l'inverse des fonctions syntaxiques définies par la théorie des kāraka ( «facteurs d'action ») par exemple, marquées morphologiquement par l'emploi de telle ou telle désinence casuelle : l'accusatif (dvitīy $\bar{a}$ ) pour l'objet (karman) (A 2.3.2 karmani dvitīya $)$, l'instrumental (tritīy $\bar{a}$ ) pour l'agent (kartro ) ou l'instrument (karana) (A 2.3.18 kartrkaranayos trtīy $\bar{a}$ ), etc. ${ }^{8}$. Comme le redira plus tard Bhartrhari, «l' lobjet de l'énoncé] n'opère pas en qualité de cause (nimitta) dans la formation des unités linguistiques [telle qu'elle se produit] au niveau des mots ${ }^{9}{ }^{9}$.

2.3. Science du mot, la grammaire n'en rencontre pas moins l'énoncé au cœur même du processus de dérivation ${ }^{10}$. Pāninini fait lui-même à deux (ou trois) reprises usage du terme vākya dans ses règles, dans des contextes bien particuliers ayant généralement trait à l'expression d'une émotion par un locuteur. Ainsi, par exemple, la règle A 8.1.8 stipule qu' « un vocatif au commencement

7. Cf. VP 2.42 : sambandhe sati yat tv anyad ādhikyam upajāyate | vākyārtham eva tam prāhur anekapadasamśrayam || «Quant à cet excès [de sens] (ädhikya), autre [que l'objet des mots], qui naît lorsque [les mots] sont mis en relation, c'est cela qu'on nomme l'objet de l'énoncé (vākyārtha), et il repose sur les mots pris collectivement ».

8. Les sections de l'Aștâahyyāȳ̄ consacrées à la théorie des kāraka (A 1.4.23-55 et 2.3.1-73) sont parmi les mieux connues de la grammaire de Pānini, nous ne nous y attardons donc pas ici. Pour un bon aperçu, on pourra consulter l'article classique de Cardona (1974).

9. Svavrtti ad VP 2.42 : na hi tasyāntahpadam <corr: antah padam Ed> śabdasamskāre nimittatvena vyāpāra äśrīyate (p. 210, 1. 22).

10. Pour davantage de détails, on se référera aux analyses très complètes de Cardona (1983: 117-127), dont nous résumons ici les principaux acquis. 
d'une phrase (vākya) (est mis deux fois ; A 8.1.1 sarvasya dve) pour exprimer l'envie, la louange, la colère, le blâme, la menace " ${ }^{11}$. C'est le cas dans l'exemple suivant :

$$
\text { arjunārjuna, śobhanah khalv asi }
$$

Ô Arjuna, que tu es beau !

Pāṇini ne semble pourtant pas considérer le terme vākya comme partie intégrante de son métalangage, dans la mesure où il n'en propose à aucun moment une définition formelle, à l'inverse du terme pada (« mot »), par exemple, qu'il définit comme " [une forme] terminée par une désinence casuelle ou personnelle » (A 1.4.14 suptinantam padam). Le besoin d'une telle définition se fait sentir dès l'époque des premiers commentateurs sur l'œuvre de Pāninini, non pas à propos des règles précitées mais afin de résoudre certaines difficultés techniques dans $l^{\prime}$ application de règles régissant l'accent $\left(\right.$ svara $\left.^{12}\right)$ et la substitution de certains pronoms personnels par des formes alternatives « brèves » (mā pour $m \bar{a} m$, te pour tava, etc. - sur ce phénomène, voir Renou, 1996 : § 253), deux thèmes traités dans le huitième et dernier livre de l'Aștādhyāy effet essentiel à la bonne application des règles régissant ces deux phénomènes qu'elles ne valent qu'«au sein d'un même énoncé » (samānavākye), comme il l'affirme dans le Vārttika suivant :

Vārttika 11 ad A 2.1.1

samānavākye nighātayușmadasmadādeśāh

La perte d'accent (nighāta) et la substitution des [pronoms personnels] yușmad (2pers) et asmad (1pers) [par des formes brèves ont lieu] au sein d'un même énoncé.

Par cette précision, Kātyāyana évite la production indue d'une perte d'accent sur la première syllabe du verbe ou la substitution erronée de tava (2pers sing, Gen) par la forme brève te respectivement dans les deux exemples suivants ${ }^{13}$ :

$$
\begin{aligned}
& \text { ayam dandah, harānena } \\
& \text { Voici un bâton, prends avec lui! } \\
& \text { odanam paca, tava bhavișati } \\
& \text { Cuis du riz, il sera pour toi! }
\end{aligned}
$$

La scission, dans les deux exemples, de la suite de mots en deux énoncés (artificiellement marquée ici par une virgule) place les deux éléments problématiques

\footnotetext{
11. A $8.1 .8:$ vākyāder āmantritasyāsūyāsammatikopakutsanabhartsaneșu. Voir aussi A 8.2 .82 : vākyasya teh pluta udāttah «(Dans les règles suivantes jusqu'à la fin du pāda, il faut entendre :) la dernière voyelle d'une phrase (tii- A 1.1.64 aco 'ntyādi ți) reçoit la pluti (protraction) et le ton aigu». La notion de $v \bar{a} k y a$ est également évoquée (quoique de manière beaucoup moins centrale) dans la règle A 6.1.139: upāt pratiyatnavaikrtavākyādhyāhāreșu; ; (L'accrément suṭ devant un $k$ - A 6.1.135 suṭ kāt pürvah - est valable entre le préverbe upa et la racine $\sqrt{ } k r-$-) pour signifier : faire effort, transformer, suppléer une proposition (vākya) (qui manque)».
}

12. La notation du sanskrit classique (i.e. autre que le védique) ignore l'accent, les réflexions des grammairiens sur ce sujet ont donc pour nous un caractère largement théorique. Tel n'était pas nécessairement le cas des premiers commentateurs sur l'Aștâadhyāylā, pour qui l'accentuation a dû encore représenter un aspect tangible de la langue dont la formalisation par la grammaire devait se conformer à un usage aujourd'hui perdu.

13. Les deux exemples sont donnés dans le Mahābhāsya ad A 2.1.1 (vol. 1 p. 367, 1. 20-21). 
(le verbe à l'impératif hara, " prends ! » et le pronom personnel tava, « à toi ») en tête de phrase, ce qui empêche l'application des règles concernées ${ }^{14}$. Reste cependant à déterminer ce qu'est un « énoncé », ce que fait Kātyāyana dans les deux Vārttika suivants (9-10), où il élabore deux définitions du vākya qui feront date :

\author{
Vārttika 9 ad A 2.1.1 \\ (K1) ākhyātam sāvyayakārakaviśeșanạ̣ vākyam \\ $\operatorname{nant}(\mathrm{s})^{15}$. \\ Vārttika 10 ad A 2.1.1 \\ (K2) ekatin \\ [L'énoncé, c'est] ce qui possède une [seule] forme conjuguée.
}

L'énoncé, c'est un verbe conjugué accompagné par un/des indéclinable(s), un/des facteur(s) d'action [i.e. noms porteurs d'une désinence casuelle] et un/des détermi-

La discussion des deux définitions de Kātyāyana et des exemples avancés à leur propos par Patañjali devait former l'ossature de la réflexion des grammairiens pāṇinéens sur l'énoncé pratiquement jusqu'à nos jours. Pourquoi, en effet, Kātyāyana a-t-il formulé deux définitions alors qu'une seule aurait pu suffire ? Le silence de Pāṇini sur la définition du vākya est-il une insuffisance de sa part ou signifie-t-il au contraire qu'une telle définition était inutile à ses yeux pour le bon fonctionnement des règles ${ }^{16}$ ? Enfin, les deux définitions formulées dans les Vārttika sont-elles propres à la grammaire et dépendantes du contexte

\begin{abstract}
14. Nous aurons à revenir (infra § 2.4) sur la règle prescrivant la perte d'accent pour une forme finie venant après une forme non finie (A 8.1.28 tin atinah). La substitution de tava par te est prescrite par la règle A 8.1.22 temayāv ekavacanasya: "Les formes te et me (sont les substituts des pronoms yușmad, <vous> et asmad, $<$ nous $>$ au génitif, datif et accusatif) du singulier ». Les deux règles en question sont gouvernées par les sūtra A 8.1.16 (padasya) et A 8.1.17 (padāt), qui établissent l'application des règles suivantes (A 8.1.18-8.3.55) à un mot (padasya) venant après un autre mot (padāt), condition que ne satisfait pas un mot placé en tête de phrase.
\end{abstract}

15. Selon l'interprétation de Patañjali les « déterminants » (viśesana) dont il est question qualifient tant les termes exprimant des facteurs d'action (kāraka) que le verbe, qui exprime l'action (kriyā). Voir Mahābhāsya ad A 2.1.1 : sakārakaviśeșaṇam. odanam mrduviśadam paca. sakriyāviśeșanam ceti vaktavyam. sușṭu pacati, dusthu pacati « Accompagné de déterminants des facteurs d'action, [comme dans l'exemple] <Il cuisine du riz tendre et qui ne colle pas $>$; mais on doit dire aussi : accompagné de déterminants de l'action, [comme dans les exemples] <Il cuisine bien> et <Il cuisine mal> » (vol. 1, p. 367, 1. 12-14). Sur la notion d' « indéclinable » (avyaya), voir Renou (1942: 70). Cette catégorie regroupe, selon Pāninini, les particules et divers autres mots invariables. Elle est définie par la règle A 1.1.37 svarādinipātam avyayam « Les mots du groupe svah (interjection rituelle) et les particules (portent le nom d')avyaya ».

16. C'est principalement ce problème qui a retenu l'attention des lecteurs modernes. Si certains - Raja (1976) et Deshpande $(1987,1991)$ - voient dans ce désaccord le signe d'une conception différente de l'énoncé chez Pānini et Kātyāyana/Patañjali, d'autres - Joshi (1968) et surtout Cardona (1983) - considèrent au contraire que le système pāṇinéen se passe d'une notion d'énoncé et dénoncent une méprise de la part de l'auteur des Vārttika concernant le champ d'application de la règle A 2.1.1 samarthah padavidhih « Une prescription visant des mots (entiers doit être comprise comme s'appliquant auxdits mots en tant que) leur sens les associe (les uns aux autres) ». Selon ces derniers, la difficulté rencontrée par Kātyāyana serait causée par l'extension indue de cette règle au-delà du deuxième livre de l'Aștâahyāy $\bar{l}$, qui traite notamment de la dérivation des composés (samāsa). Dans cette hypothèse, la réflexion sur la notion d'énoncé perd sa pertinence dans le cadre dérivationnel et se trouve exclue du champ d'investigation de la grammaire telle que le conçoit le Vyākarana. 
technique qui les occasionne, ou peuvent-elles valoir comme définitions générales de l'énoncé ? Retenons pour le moment que les deux définitions avancées par Kātyāyana dégagent certaines propriétés formelles de l'énoncé sans tenir compte de ce qu'il exprime : ce qui caractérise un vākya c'est soit la centralité fonctionnelle du verbe, dont tous les autres éléments (indéclinables, facteurs d'action, etc.) dépendent, soit l'unicité de la forme conjuguée.

2.4. Cette dernière caractéristique, sur laquelle tous les commentateurs s'accordent (sinon sur laquelle des deux définitions en fait état !) permet de mettre en lumière un dernier aspect de la réflexion de Kātyāyana sur l'énoncé, à savoir sa portée polémique à l'encontre de Pānini. Cette dimension est clairement perceptible lorsque Kātyāyana se penche à nouveau, dans ses remarques sur le huitième livre de $l^{\prime} A s t a \bar{a} d h y \bar{a} y \bar{\imath}$, sur la principale règle prescrivant la perte de l'accent (nighāta) sur la première syllabe d'une forme conjuguée :

\section{A 8.1.28}

tin ativiah

Une forme conjuguée (perd son accent - A 8.1.18 anudāttam sarvam apādādau) après un mot qui n'est pas une forme conjuguée ${ }^{17}$.

En effet, la formulation de cette règle, et notamment la précision atinah ( «après un mot qui n'est pas une forme conjuguée »), ne se justifie que dans l'hypothèse où un énoncé possède plus d'une forme conjuguée (tii). Or, c'est là précisément ce qui est impossible dans la conception du vākya développée par Kātyāyana, lequel adresse à Pānini une critique (pratyākhyāna) tout à fait explicite :

Vārttika 1 ad A 8.1.28

ativivacanam anarthakam samānavākyādhikārāt

[Dans la règle 8.1.28 tin atinah] la mention atinah (" après un mot qui n'est pas une forme conjuguée ») est inutile, car [la règle] est gouvernée par [la condition] samānavākye ("au sein d'un même énoncé »).

Comme le précise Patañjali, le problème est ici que « deux formes conjuguées ne peuvent se trouver au sein d'un même énoncé » (na [...] samānavākye dve tinante stah - Mahābhāṣya ; vol. 3, p. 374, 1. 25) ; il est donc impossible qu'une forme conjuguée suive une autre forme conjuguée.

2.5. Par-delà l'enjeu restreint que constitue l'application des règles d'accentuation, on voit se dessiner, à travers ce débat entre les deux plus anciennes autorités de la grammaire pāninéenne, l'opposition entre deux conceptions de l'énoncé : l'une, celle de Kātyāyana, insiste sur son unité fonctionnelle, centrée sur l'existence d'un unique verbe conjugué ; une autre, que l'on ne se hâtera pas trop d'attribuer à Pānini, néglige ces caractéristiques de surface au profit de l'unicité de l'acte d'énonciation et de son intention, indépendamment de sa forme linguistique externe. Ce n'est qu'avec Bhartrhari, et par un contact direct

17. Nous modifions légèrement la traduction de Renou (1966 : 358) : «Une forme verbale personnelle (a le ton grave, venant) après un mot qui n'est pas une forme personnelle ». 
avec la tradition exégétique, que ces deux conceptions opposées devaient venir pleinement au jour.

\section{BHARTŖHARI : L'ÉNONCÉ ENTRE GRAMMAIRE ET EXÉGÈSE}

3.1. Composé quelque sept siècles après le Grand Commentaire de Patañjali sur l'Aștādhyāy $\bar{\imath}$, le Vākyapadīya de Bhartrohari témoigne du caractère remarquablement florissant de la réflexion sur l'énoncé dans l'école pāṇinéenne au milieu du premier millénaire, une floraison dont il constitue cependant l'une des rares traces. Les deux stances programmatiques ouvrant la "Section sur l'Énoncé » (Vākyakānda) - la seconde partie de l'ouvrage, qui en compte trois - n'énumèrent en effet pas moins de huit théories du vākya, examinées par Bhartrhari dans la suite du chapitre ${ }^{18}$ :
VP 2.1-2
ākhyātam śabdasamghāto jātih samghātavartin̄i |
eko 'navayavah śabdah kramo buddhyanusamhrtih || 2.1 ||
padam àdyam prothak sarvam padam sāpekșam ity api |
vākyam prati matir bhinnā bahudhā nyāyadarśinām || 2.2 ||
(1) Un verbe (ākhyāta), (2) un agrégat (samghāta) de signes linguistiques (śabda),
(3) l'universel (jāti) présent dans [cet] agrégat, (4) un unique signe linguistique (śabda) sans parties (anavayava), (5) la séquence (krama) [des signes / des mots], (6) le rassem- blement [de la série signifiante] par la pensée (buddhyanusamhrrti ${ }^{19}$ ), (7) le premier mot [de la série] (padam ādyam), (8) chaque mot pris séparément (prthak), dépendant [des autres mots] (sāpekșa) : l'idée [que se font] les théoriciens de l'énoncé (vākya) diffère de bien des manières!

L'origine de la plupart des conceptions de l'énoncé énumérées dans ces stances n'est pas connue et leur teneur même n'est pas toujours parfaitement claire ${ }^{20}$, une situation que n'améliore pas l'absence de l'autocommentaire de Bhartrhari sur la section initiale du Vākyakānda (VP 2.1-12). On reconnaîtra cependant sans peine dans la première de ces huit définitions la première définition de Kātyāyana (K1), identifiant l'énoncé avec un (unique) verbe conjugué (ākhyāta) accompagné d'éléments auxiliaires (déterminants, facteurs d'action, etc.). De fait, c'est bien le débat sur les définitions du vākya tel qu'on l'a vu s'élaborer chez Kātyāyana et Patañjali qui constitue le point de départ des réflexions de Bhartrhari dans ce chapitre. La question de la définition de

\footnotetext{
18. Les stances du Vākyapadīya sont citées d'après l'édition critique de Rau (1977). La numérotation des stances suit cependant l'édition d'Iyer (1983) qui comprend, outre les stances du VP, l'autocommentaire (Svavrtti) de Bhartrọhari et un commentaire plus tardif sur le second kānḍa, la Tīkā de Punyarāja (début du deuxième millénaire?).
}

19. Le terme buddhyanusamhrti / ${ }^{\circ}$ anusamhāra [VP 3.9.57c, VPSV 2.30] (« rassemblement par la pensée ») désigne pour Bhartrihari la saisie par l'esprit d'une séquence (dans le cas présent, d'une séquence verbale) dans une cognition instantanée.

20. Sur les hésitations des auteurs indiens médiévaux concernant l'interprétation de cette liste, voir les remarques de Raja (1962). 
l'énoncé (vākyalakṣana) est abordée une première fois dans les stances immédiatement subséquentes (VP 2.3-6), dans lesquelles Bhartrorhari discute la première définition des Vārttika (K1). Elle est à nouveau évoquée vers la fin du chapitre (VP 2.442-446 et Svavrtti) ; c'est alors la seconde définition de Kātyāyana (K2) qui passe au premier plan, ainsi que la critique qu'il adresse à Pānini. C'est à l'analyse de ces deux groupes de stances qu'est principalement consacrée la présente section ${ }^{21}$.

3.2. Bhartrarhari entame sa réflexion sur les définitions du vākya par une stricte délimitation du domaine de validité des définitions de Kātyāyana. Celles-ci ne sauraient en effet, selon lui, constituer une définition générale de l'énoncé, mais servent exclusivement à la bonne application des règles grammaticales d'accentuation, etc. :

VP 2.3-4

nighātādivyavasthārtham śāstre yat paribhāṣitam |

sākāinkṣāvayavam tena na sarvam tulyalakșanam || 2.3 ||

sākāinkṣāvayavam bhede parānākāinkșaśabdakam |

karmapradhānam gunavad ekārtham vākyam ucyate || 2.4 ||

[L'énoncé ${ }^{22}$ tel qu'il est] techniquement défini (paribhāșita) dans la grammaire afin d'assurer l'application correcte de la perte d'accent (nighāta) et autres [phénomènes semblables] ne partage pas toutes les caractéristiques de [l'énoncé] défini comme cela dont les membres sont en attente [d'autre chose lorsqu'ils sont séparés] (2.3). On dit [en effet] que l'énoncé est [un signe linguistique] dont les membres sont en attente [d'autre chose] lorsqu'ils sont séparés, composé de signes qui ne sont en attente de rien d'autre ${ }^{23}$, dont l'élément principal est l'action accompagnée d'auxiliaires, et dont l'objet (artha) est unique ${ }^{24}(2.4)$.

Bhartrọhari oppose ici à la définition technique (*pāribhāṣika / *śāstrīya) de l'énoncé par Kātyāyana, une autre définition qu'il emprunte à la Mìmāmsāa, l'éxégèse du Veda. Les exégètes avaient en effet développé depuis l'époque des Mìmāmsāsūtra ("Aphorismes sur l'exégèse ») une réflexion indépendante sur l'énoncé, partant de problèmes tout à fait différents. Il s'agissait avant tout, pour ces interprètes du Veda dans une perspective ritualiste, de trouver un critère

21. Sur VP 2.3-4, on pourra consulter l'article en japonais de Honda (1996). La Svavrtti sur VP 2.442 est discutée, quoique fort brièvement, par Houben (1998 : 126-127).

22. Nous suppléons ici le sujet vākyam («l'énoncé »), repris de la stance précédente (VP 2.2cd, traduite supra § 3.1). Cette interprétation va à l'encontre du commentaire de Punyarāja, qui considère que le sujet est plutôt vākyalaksanam (« la définition de l'énoncé »-Ṭ̄kā ; p. 5, 1. 25-27). Elle est cependant confortée par une remarque de Kaiyata, qui paraphrase Bhartrhari dans le Pradīpa (vol. 2, p. 336b, 1. 14-15) et affirme que « l'énoncé fait l'objet d'une définition technique (vākyam paribhāsyate) afin d'éviter qu'on n'utilise la définition profane (laukika) de l'énoncé [dans l'application du Vārttika 11, samānavākye, etc.] » (laukikavākyagrahananiṣedhārtham vākyam paribhāsyate ; sur la règle en question, voir supra $\S 2.3$ ).

23. Entendons : de rien qui serait extérieur à l'énoncé.

24. Nous maintenons volontairement dans notre traduction l'ambiguïté du terme sanskrit artha (« objet»), qui désigne à la fois la « référence » d'un signe linguistique et le « motif » de son énonciation. Punyarāja, s'appuyant vraisemblablement sur le Śäbarabhāsya (vol. 2 p. 436, 1. 2), mentionne uniquement le second sens (ekārtham ekaprayojanam - Tīkā ; p. 6, 1. 9). 
efficace leur permettant de délimiter au sein du continuum du texte védique les formules sacrificielles (yajus) destinées à être récitées par les officiants au cours d'un rite ${ }^{25}$ :

Śābarabhāṣya ad Mìmāmsāsūutra 2.1.46 (vol. 2, p. 432, 1. 1)

atha praślișțapaṭiteșu yajuḥsu katham avagamyata iyad ekam yajur iti

À présent [on considère le problème suivant]: puisque les formules sacrificielles (yajus) sont récitées de manière conjointe (praślișta), comment comprend-on qu'une formule sacrificielle a précisément telle ou telle extension?

Si cette préoccupation croise de toute évidence celle des grammairiens - puisqu'il s'agit toujours finalement de délimiter l'énoncé en définissant son unité (ekavākyatā) -, la solution de Jaimini est différente de celle de Kātyāyana en ce que l'unité du vākya est déterminée, selon lui, par un critère sémantique et non plus formel :

\section{Mìmāmsāsūtra 2.1.46 \\ arthaikatvād ekạ̣ vākyam sākānikṣam ced vibhāge syāt \\ Un énoncé est unique si son objet est unique et si [ses parties] sont en attente [d'autre chose] lorsqu'elles sont séparées.}

Les exégètes ne s'accordent pas sur la portée exacte de cette règle, qui définit tant le vākya lui-même que son unité. Si les auteurs anciens considèrent qu'elle s'applique uniquement au yajus ${ }^{26}$, certains adeptes de la Mìmāmssā plus tardifs acceptent sa valeur pour d'autres types d'énoncés védiques, voire pour tout énoncé quel qu'il soit ${ }^{27}$. Telle semble être également la position de Bhartroari, lequel adopte presque sans changement la définition du sūtra en y ajoutant simplement deux critères supplémentaires : la centralité de l'action et l'absence d'attente (anākāinkṣ $[\bar{a}]$ ) d'éléments extérieurs à l'énoncé ${ }^{28}$. C'est avant tout la conception de l'énoncé comme entité dynamique régie par un réseau d'attentes $(\bar{a} k \bar{a} \dot{n} k s ̣ \bar{a})$ sémantiques et syntaxiques qui semble intéresser le grammairien, qui fera dans la suite du Vākyakāṇda régulièrement appel à la notion d'a $k \bar{a} n \dot{i} k s \bar{a}^{29}$.

\footnotetext{
25. Dans la terminologie du rituel védique, les yajus sont des formules (mantra) récitées à voix basse par le prêtre adhvaryu, chargé des opérations rituelles proprement dites (oblations, aspersions, etc.). Ils se distinguent des $r c$ («strophes ») récitées à voix haute par le prêtre hotr et des sāman (« chants ») entonnés par le chantre (udgātr). Contrairement aux formules de ces deux dernières catégories, qui sont versifiées et dont la délimitation ne pose donc pas problème, les yajus sont en prose et ne peuvent aisément être isolés lors d'une récitation continue (le texte du Veda étant, comme on sait, principalement transmis sous sa forme orale à l'époque qui nous intéresse).
}

26. Ce point fait notamment l'objet d'un long développement par Kumārila (VI ${ }^{\mathrm{e}}-\mathrm{VII}{ }^{\mathrm{e}}$ s.) dans le Tantravārttika (vol. 2, p. $431,1.15-$ p. $434,1.26$ ).

27. C'est par exemple l'opinion de Bhavadeva (XII s.) et Khaṇdadeva (XVII ${ }^{\mathrm{e}}$ s.). Voir Tautātitamatatilaka (Bhavadeva, p. 298, 1. 5-6) et Mìmāmsākaustubha (Khaṇḍadeva, p. 120, 1. 12-16).

28. Ce dernier critère pourrait s'inspirer de réflexions ritualistes antérieures aux Mīmāmsāsūtra. Voir par exemple Kātyāyanaśrautasūtra (1.3.2) : teșām vākyam nirākāìkṣam « Pour ce qui est des [formules sacrificielles, yajus], un énoncé est ce qui est dépourvu d'attente ».

29. Voir notamment VP (SV) 2.9, 76, 350, 426-27, 442 (cité § 3.3) et 445 (cité infra). 
Bhartrhari s'efforcera alors d'affiner cette notion en distinguant $l^{\prime}$ « attente verbale » (śabdalakșanākāinkșā) - syntaxique en d'autres termes - qui fait qu'une forme nominale à l'accusatif, par exemple, appelle un verbe ou un participe, $\mathrm{d}^{\prime}$ une attente « objective » (arthalaksan $a^{\circ}$ ) portant davantage sur les éléments de contexte que l'auditeur pourrait souhaiter connaître. La Svavrtti sur VP 2.445 (Rau 2.450) évoque à ce propos l'exemple suivant (p. 316, 1. 4) :

vrīhīn avahanyantām

"Qu'on effectue le battage [de manière à séparer] les grains de riz »

L'énoncé (5), prescrivant le battage du riz préalable à la confection de gâteaux offerts au cours du rite, est "sans attente » (anākāinkșa / nirākānikșa) en ce qu'il ne requiert pour remplir sa fonction l'adjonction de rien d'autre, à l'inverse d'un énoncé tronqué comme vrìhìn ( « les grains de riz»), lequel ne communique rien à moins qu' on ne lui adjoigne un verbe, un participe, etc. L'auditeur pourra bien entendu s'interroger plus avant, par exemple, sur les modalités de l'action prescrite : comment se fait le battage, à quel moment, avec quels instruments, etc. ? Ce second type d'attente, " objective » en ce qu' elle porte sur la situation concrète dans laquelle la phrase est prononcée, virtuellement infinie et étroitement dépendante des désirs de l'auditeur et de son savoir préalable, n'intervient cependant pas pour définir la complétude de l'énoncé, comme le remarque Bhartroari dans la stance correspondante :
VP 2.445 (Rau 450)
itikartavyatārthasya sāmarthyād yatra kāikssyate
aśabdalakṣanākāinkṣam samāptārtham tad ucyate || 450 ||

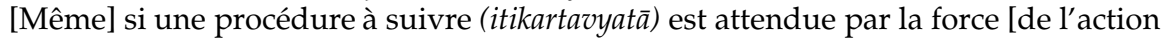 prescrite], on dit que [l'énoncé] est complet du point de vue de son objet (samāptārtha) s'il est libre d'attente verbale (aśabdalakșaṇākāinksa).

3.3. L'emprunt massif par Bhartronari de concepts élaborés par les théoriciens du rituel devait le conduire à une discussion critique avec Kātyāyana, ainsi qu'à une relecture en profondeur de son conflit avec Pāninin. C'est en effet le critère même de l'unité de l'énoncé selon l'auteur des Vārttika - l'unité du verbe conjugué (ākhyāta/tin) - qui se trouve chez lui remis en cause au nom d'une unité plus fondamentale :

VP 2.6

yathānekam api ktvāntam tinantasya viśeșakam

tathā tinantạn tatrāhus tinantasya viśeșakam \|

On affirme que, tout comme dans un [même énoncé] plusieurs absolutifs (ktvānta) peuvent qualifier une forme conjuguée (tinanta), de même une forme conjuguée peut qualifier une [autre] forme conjuguée.

Bhartrori ne donne malheureusement aucun exemple de phrase où ce principe $\mathrm{s}^{\prime}$ appliquerait, on est donc contraint de s'en remettre aux commentateurs. Parmi eux, Punyarāja est à ma connaissance le seul à évoquer les conséquences proprement grammaticales de la remarque de Bhartrohari, et considère à ce propos l'exemple suivant $(T i \bar{k} k \bar{a}, \mathrm{p} .7,1.8)$ : 
(6)

pūrvam snāti(,) pacati tato(,) vrajati tatah

D'abord il se lave, puis il fait la cuisine, puis il sort.

Cette phrase se caractérise par la présence de trois formes conjuguées : snāti (« il se lave »), pacati (« il fait la cuisine ») et vrajati (« il sort »). Or, l'usage - autorité ultime en matière d'accentuation - prescrit la perte d'accent (nighāta) sur la première syllabe du verbe vrajati, justifiée dans la dérivation par l'application de la règle $\mathrm{A}$ 8.1.28 tin atinah (cf. supra $\S 2.4$ ). La division de la chaîne signifiante en trois vākya (marqués ici par des virgules) suivant le critère fixé par Kātyāyana (cf. supra § 2.3) empêcherait cependant l'application de la règle pānininéenne au verbe vrajati, placé en tête de phrase en vertu de cette division. Il est donc nécessaire, pour rendre compte de cet exemple, soit d'abandonner la clause selon laquelle la règle ne s'applique qu'au sein d'un même énoncé (samānavākye) ce qu'à notre connaissance aucun auteur postérieur à Kātyāyana ne fait -, soit d'ajuster la définition grammaticale du vākya de sorte qu'elle puisse s'accommoder aussi d'un énoncé contenant plus d'un verbe conjugué. C'est ce que fait Bhartrhari vers la fin du chapitre, lorsqu'il aborde une seconde fois la question des définitions grammaticales de l'énoncé :

\begin{abstract}
VP 2.442-443 (Rau 2.447-448)
bahuṣv api tinanteșu sākāinkṣeșv ekavākyatā |

tināạm ${ }^{30}$ tinbhyo nighātasya paryudāsas tathārthavān \|

ekatin yasya vākyam tu śāstre niyatalakṣanam |

tasyātingrahanenārtho vākyabhedān na vidyate \|

Même si plusieurs verbes conjugués [se succèdent], il s'agit d'un unique énoncé s'ils sont en attente (sākāinkșa) [les uns des autres] ; de la sorte, l'exclusion [par Pāṇini] de la perte d'accent pour les formes conjuguées [venant] après [d'autres] formes conjuguées [en A 8.1.28 tin atinah] est pleinement justifiée. Mais si l'on maintient [avec Kātyāyana] que l'énoncé est défini de manière constante (niyatalakșaṇa) dans la grammaire comme ce qui possède une [seule] forme conjuguée (ekatin), il n'y a aucune raison de préciser atinah (" après une [autre] forme verbale »), car il s'agit [dans ce cas] d'une pluralité d'énoncés (vākyabheda).
\end{abstract}

Bhartrohari propose dans ces stances une solution inédite au conflit entre Pānini et Kātyāyana : si Pānini exclut dans le sūtra 8.1.28 la perte d'accent pour une forme finie suivant une autre forme finie, c'est qu'il avait de l'énoncé une conception plus large que celle de Kātyāyana, autorisant qu'une phrase possède plus d'une

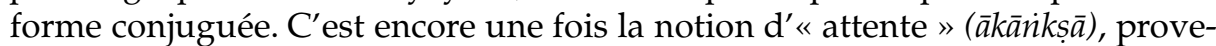
nant comme on l'a vu de la réflexion des exégètes, qui permet à Bhartrohari d'expliciter cette conception soi-disant « pāṇinéenne » du vākya que l'on chercherait évidemment en vain dans $l^{\prime} A s \underline{t} \bar{a} d h y \bar{a} y \bar{\imath}$ : plusieurs verbes conjugués forment un seul énoncé s'ils sont « en attente » (sākāinkṣa) les uns des autres. Comme le précise la Svavrtti, il ne peut cependant plus s'agir comme supra (§ 3.2) d'une attente de type « verbal » (śabdalakṣana - un verbe conjugué ne nécessitant jamais d'un point de vue syntaxique la présence d'un autre verbe conjugué -, mais d' « une

30. Nous corrigeons ici le texte de Rau (1977: 100), qui lit tinā tinbhyo. Cf. Houben (1998: 126). 
certaine attente objective " (arthalakṣaṇa kā cid ākāinkṣā; p. 315, 1. 10) due à la présence d'une unité supérieure, d'ordre sémantique, à savoir " l'unité d'objet » (ekārtha[tva]) dont parlait Bhartronari dans sa reformulation de l'aphorisme de Jaimini. Par le biais d'un emprunt à la Mìmāmssā, Bhartronari peut ainsi se présenter paradoxalement, contre Kātyāyana, comme le défenseur d'une certaine orthodoxie pāninéenne.

\section{CONCLUSION}

Par sa réflexion sur les définitions du vākya, Bhartrorhari réconcilie ainsi la préoccupation de Kātyāyana et Patañjali pour l'application des règles dans la dérivation avec une réflexion philosophique plus large sur l'énoncé. Cette dernière, qui occupe toute la seconde partie de son œuvre, trouve son sommet dans sa conception de l'énoncé comme entité « indivise » (akhanda), qualitativement distincte des éléments qui le composent. C'est vers cette unité close sur elle-même, dans laquelle il n'est plus possible de distinguer (sinon pour les besoins de l'analyse) aucun « signe » ou « mot » distinct du tout que tend l'analyse bhartrharienne du vākya comme entité « sans attente » (nirākānikṣa), régie par l'unité d'une intention ${ }^{31}$. La tradition grammaticale plus tardive tendra à négliger cette perspective et adoptera, de ce fait, une attitude ambivalente vis-à-vis des positions de Bhartrhari. Ainsi Kaiyața (Cachemire, $\mathrm{XI}^{\mathrm{e}} \mathrm{s}$.), l'auteur du plus célèbre commentaire sur le Mahābhāsya, le Pradīpa ( Lumière [sur le Mahābhāṣya] »), doit-il encore beaucoup à l'auteur du Vākyapadīya, à qui il emprunte notamment une interprétation littérale, et purement instrumentale pour la dérivation, des définitions des $V \bar{a} r t t i k a$. La situation change radicalement à l'époque des sous-commentaires sur le Pradīpa. Tant Śivarāmendra Sarasvatī (seconde moitié du XVII ${ }^{\mathrm{e}} \mathrm{s}$.) que Nāgeśa Bhatța (XVIII $\mathrm{e}$ s.) - pour ne citer que les plus célèbres - avancent en effet contre Kaiyața (et à travers lui contre Bhartronari) une défense intransigeante des positions de Kātyāyana, interprétées au besoin de manière non littérale. Malgré l'indéniable ingéniosité des commentateurs dans leur défense des définitions canoniques, ce repli de la tradition grammaticale sur elle-même et ses plus anciennes autorités marque un certain appauvrissement de la discussion sur l'énoncé, conséquence du relatif émoussement de l'influence de Bhartrọari au profit des seuls « Trois muni ». Ainsi l'énoncé devait-il, après un bref âge d'or au premier millénaire, se trouver à nouveau exclu du champ de la grammaire pāṇinéenne.

\footnotetext{
31. Ce point devient parfaitement clair dès la première formulation de la thèse bhartraharienne de l'énoncé « indivis » (akhanda) dans les stances 7-10 du Vākyakānda, où l'on retrouve sans surprise la notion d'un énoncé « de toutes parts sans attente » (nirākäriksa[h] sarvatah ; VP 2.9b).
} 


\section{Références}

\section{Sources primaires}

BHARTRHARI, Vākyapadīya (VP). [voir Rau 1977]

BHARTRoHARI, Vākyapadiya-Svavirtti (VPSV) et Vākyapadīya-Ṭikā de Punyarāja (kāṇda 2), Delhi/Varanasi/Patna : K.A. Subramania lyer, 1983.

BhAVADEVA, Tautātitamatatilaka, Bénarès : A. Cinnasvāmi Śāstrī \& P. N. Patțābhirāma Śāstrī, 1999.

KaIYAṬA, Pradīpa, dans Vyākaranamahābhāṣya, vol. 2, Delhi : Pandit Shivdatta Sharma, 1988. [reprint]

KĀTYĀYANA, Vārttika. [voir Patañjali, Mahābhāṣya]

Kātyāyanaśrautasūtra, Bénarès : A. Weber, $1972^{2}$.

KhaṇḍadeVA, Mīmāṃsākaustubha, Bénarès : A. Cinnasvāmi Śāstrī, 1991.

KUMĀRILA BHAṬ̣A, Tantravārttika. [voir Mīmāmsāsūtra]

Mīmāmsāsūtra attribués à JAIMINı (vol. 2), avec le Bhāṣya de ŚABARA et le Tantravārttika de KumĀRILA BHAṬTA, Pune : Gaṇeśaśātrī Jośī, 1981³.

PĀṆINI, Așțādhyāyì (A). [voir Renou 1966]

PatañJalı, Mahābhāṣya, 3 vol., Pune : F. Kielhorn, rév. K.V. Abhyankar, 1985 . [voir aussi Joshi 1968]

PUṆYARĀJA, Vākyapadīya-Ṭikā. [voir Bhartṛhari, Vākyapadìya-Svavrtti]

Śābarabhāṣya = Mīmāṃsāsūtrabhāṣya de ŚABARA(SVĀMIN). [voir Mīmāṃsāsūtra]

\section{Études et traductions}

CARDONA G. (1974), "Pānini's kārakas: agency, animation and identity”, Journal of Indian Philosophy 2, 231-306.

CARDONA G. (1983), "Some questions regarding the sentence and its constituents", Linguistic Analysis and Some Indian Traditions, Poona: Bhandarkar Oriental Research Institute, 117159.

DAVID H. (2016), "Time, action and narration: on some exegetical sources of Abhinavagupta's aesthetic theory", Journal of Indian Philosophy 44 (1), 125-154.

DeSHPANDE M. M. (1987), "Pāninian syntax and the changing notion of the sentence", Annals of the Bhandarkar Oriental Research Institute 68, 55-98.

DeshPANDE M. M. (1991), "Pānininian syntax and the changing notion of the sentence", in $\mathrm{H}$. Hock (ed.), Studies in Sanskrit Syntax. A volume in Honor of the Centennial of Speijer's Sanskrit Syntax (1886-1986), Delhi: Motilal Banarsidass, 31-43.

HoNDA Y. (1996), “Vākyapadīya, Chpt. II, kk. 3-4 - on 'vākya' Definition”, Journal of Indian and Buddhist Studies 44 (2), 913-910 [68-71].

HOUBEN J. (1998), “The theoretical positions of Bhartrhari and the respectable grammarian”, Rivista degli studi orientali 72, 101-141.

JosHı S. D. (1968), Patañjali's Vyākarana-Mahābhāṣya. Samarthāhnikah (P 2.1.1), Poona: University of Poona.

MCCREA L. J. (2008), The Teleology of Poetics in Medieval Kashmir, Cambridge (MA): Harvard University Press.

RAJA K. K. (1962), “Bhartrhari's list of sentence-definitions”, Adyar Library Bulletin 26, 206-210. RAJA K. K. (1976), “Pāṇini's concept of the sentence”, Adyar Library Bulletin 40, 165-170. 
RAU W. (1977), Bhartroharis Vākyapadīya, Wiesbaden : Franz Steiner.

RENou L. (1942), Terminologie grammaticale du sanskrit, 3 vol., Paris : Honoré Champion.

Renou L. (1966), La grammaire de Pāninini, 2 vol., Paris : École française d'Extrême-Orient.

RENOU L. (1996), Grammaire sanskrite, Paris : Adrien Maisonneuve. 